\title{
Research and Experimental Verification of the 126kV Vacuum Interrupter Contact Structure
}

\author{
Xue Congjun ${ }^{1, a}$, Li Xiaozhao ${ }^{1, b}$, Liu Chang ${ }^{1, c}$, Liu Shibai ${ }^{1, d}$, Zhao Fangshuai1, e, \\ Pu Liangjing ${ }^{1, f}$ \\ ${ }_{1}^{1}$ Ping Gao Group Co., Ltd. Pingdingshan, 467001, China \\ axcj329@126.com, blixzpg@126.com, ‘6379468@qq.com, bliusbpg@126.com, , \\ ezhaofspg@126.com, ${ }^{\dagger}$ puljpg@126.com
}

Keywords: Contact structure; $126 \mathrm{kV}$ vacuum interrupter;AMF; simulation analysis

Abstract. in this paper, the magnetic field and arc of a contact structure of $126 \mathrm{kV}$ vacuum interrupter are studied and tested. The magnetic field values of the contact structure is measured and compared with the results of simulation. The results of simulation are verified by comparing the results of the two calculations. The behaviors of vacuum arc of the contact structure was studied by means of arcingexperiment, The $126 \mathrm{kV}$ vacuum interrupter is manufactured by this contact structure, and the 40kA short-circuit current breaking test is carried out. After test and verification, the vacuum interrupter can break 20 short-circuit currents of $40 \mathrm{kA}$ and analyze the state of the vacuum interrupter after the test. Through a series of research and verification, it is proved that this vacuum interrupter contact structure can complete the breaking performance requirements of the $126 \mathrm{kV}$ vacuum interrupter.

\section{Introduction}

Due to the excellent insulation and arc extinguishing characteristics of vacuum circuit breaker, vacuum circuit breaker has become the mainstream and future development direction in the field of medium voltage switches ${ }^{[1][2]}$. In the field of high voltage, the $126 \mathrm{kV}$ and above switchgear used in power transmission and distribution systems are all oil switches or $\mathrm{SF}_{6}$ switches. As the dominant one, SF6 circuit breakers are also used. $\mathrm{SF}_{6}$ circuit breakers use SF6 gas as the arc extinguishing medium. However, because $\mathrm{SF}_{6}$ is a greenhouse gas, its greenhouse effect is 23,900 times that of $\mathrm{CO}_{2}$. the international vacuum switch field is very concerned about the development of the vacuum switch from the medium voltage level to the higher voltage level occupied by the SF6 circuit breaker ${ }^{[3-5]}$. The structure of the contact is the most important factor affecting the breaking capacity of the vacuum circuit breaker ${ }^{[6][7]}$.

To improve the breaking capacity of the vacuum interrupter, it is necessary to thoroughly study the influence of the axial magnetic field (AMF) on the vacuum arc, Find out the law of AMF acting on the vacuum arc, and then take measures to improve the contact structure of the vacuum interrupter, in order to make better use of the characteristics of AMF, reduce the energy of the arc and improve the breaking capacity of the vacuum interrupter ${ }^{[8][9]}$, Based on previous research, at present the contact structure of the $126 \mathrm{kV}$ vacuum interrupter is mainly composed of two kinds of coil structure and horseshoe structure, both of which are the AMF contact structures. Both have their own advantages and disadvantages, but the structure of the coil type contact is more is more advantageous in the field of large current breaking ${ }^{[10]}$.

\section{$126 k$ New AMF Contact Structure}

The new AMF contact structure for $126 \mathrm{kV}$ vacuum interrupter is shown in Fig. 1. the upper end is a static contact structure, the lower end is a dynamic contact structure. The overall contact structure

This work is supported by State grid headquarters' science and technology projects

consists of conductive rods, coils, and contact pieces. The portion between the static and dynamic contacts is the arc region. In Figure 1, the arrow indicates the path of the current. Each contact coil has 
two layers of coils, namely, two upper and lower layers. and the contact pieces are respectively connected in parallel by two branches. The height and width of the upper and lower coils are the same, and the magnetic field is mainly generated by the coils of the dynamic and static contacts. The overall layout of the dynamic and static contact structure is shown in Fig. 1. With this structure, the magnetic field in the entire arc region is produced by the current in the three-layer coil, which ensures that the magnetic field value in the arc region is the largest, and the distribution is even, avoiding the concentrated ablation of contacts by arc during breaking process. As shown in Figure 2, each conductor loop corresponds to a circumference of $270^{\circ}$, the contact diameter is $98 \mathrm{~mm}$, the coil width is $10 \mathrm{~mm}$, and the coil height is $10 \mathrm{~mm}$. The value of the current flowing through the two branches of the coil is the same, which is $1 / 2$ of the total current value. Current path: Through the conductive rod of static contact, enters the coil and then enters the contact chip, the movable contact is opposite, the current passes through the contact chip into the coil, and then the conductive rod. Contact blade diameter is $100 \mathrm{~mm}$, open four slots as shown in Figure 1, reduce eddy currents, helps reduce residual magnetic field after current zero crossing, the smaller the residual magnetic field, the more conducive to the recovery of the dielectric strength, it is more conducive to the circuit breaker Breaking.

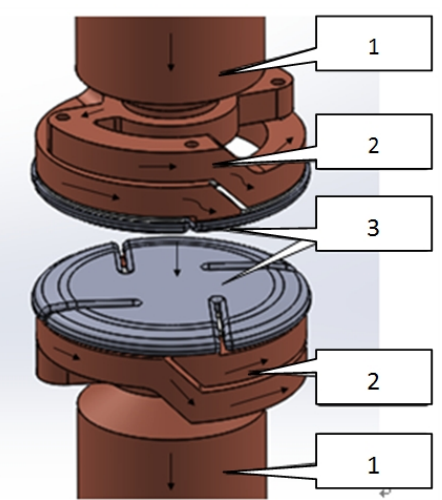

Fig.1 Contact Structure Layout

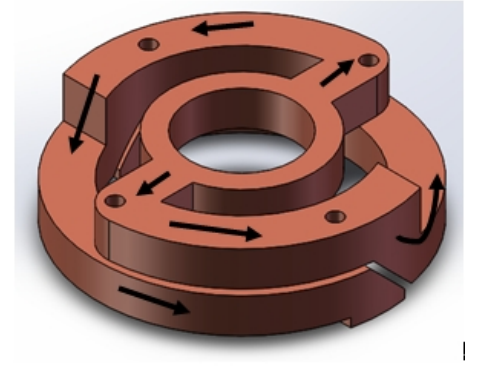

Fig.2 New Structure of AMF Coil

1-Contact rod; 2-coil;3-Contact plate

\section{Simulation Analysis of Magnetic Field Characteristics}

Using simulation analysis and experimental method, verify the magnetic field characteristics of the contacts.

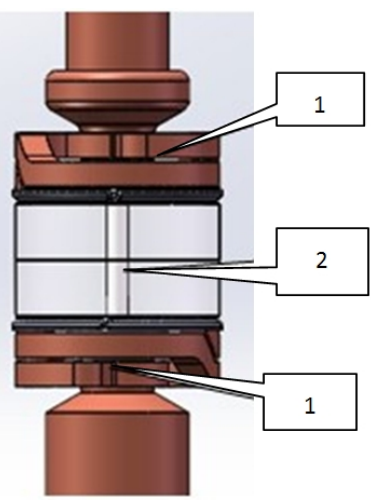

Fig.3 simulation model of coil structure 1-Coil; 2-Arc

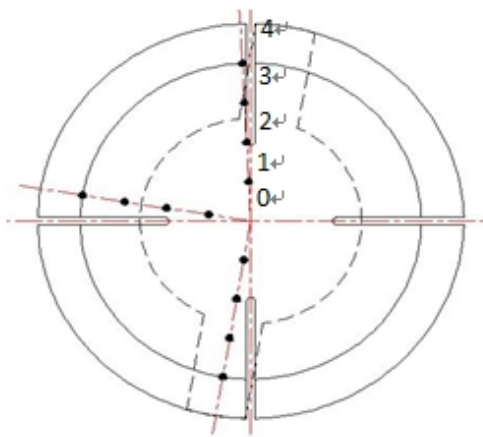

Fig.4 the extraction position of the magnetic field value

The simulation calculations use electromagnetic field software Maxwell Ansoft. The simulation model is shown in Fig. 3. The rated clearance between open contacts is $55 \mathrm{~mm}$. The magnetic field value extraction is shown in Fig. 4. The simulated arc is placed at point 0 in the figure, and points 1, 2, 3 , and 4 are the extracted magnetic field values of the coil. In the magnetic field measurement experiment, the contact structure was fixed in the tooling, the material of the tool was made of Teflon 
(nonconductive non-magnetic), and the diameter was $8 \mathrm{~mm}$. The length of the 55mm copper rod (arc) was used as the conduction channel in the middle of the two contact pieces.

Simulation calculations, loading DC 300A, and the magnetic field values of 4 points in Figure 4 were extracted. The magnetic field values of 1-4 points are shown in Figure 5; In the measurement experiment, the input DC 300A, the clearance between open contacts $55 \mathrm{~mm}$, and magnetic field values of 1-4 points are shown in Figure 6. The unit of magnetic field: mT

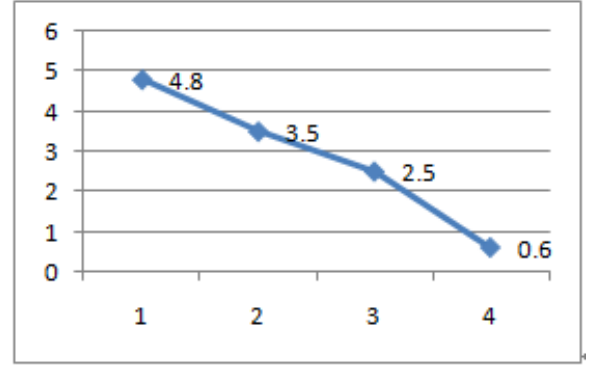

Fig.5 magnetic field value

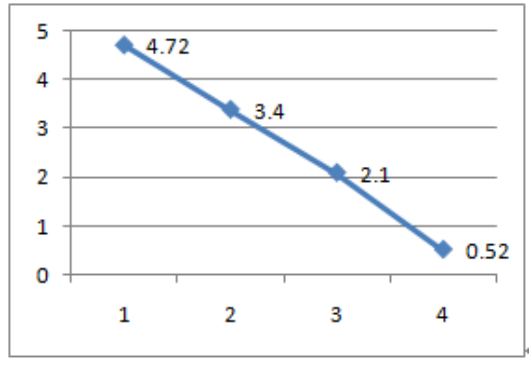

Fig.6 magnetic field value

Loading AC 300A, to extract the magnetic field value and the magnetic field lag time at the current of 0 for 4 pointsin FIG. 4 . In the measurement experiment, loading AC 300A, when current is zero, the magnetic field size and magnetic field lag time, as shown in Figure 7-10. The unit of magnetic field: Mt, Time unit: $\mu \mathrm{S}$

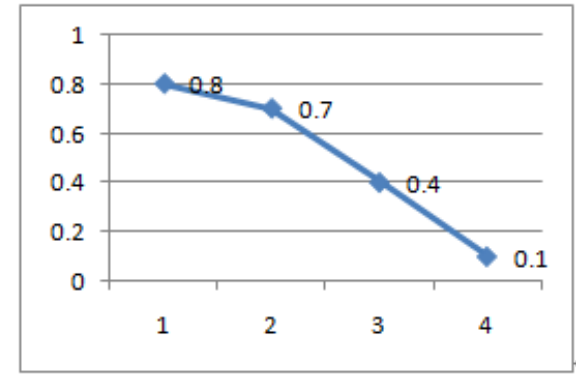

Fig.7 magnetic field value

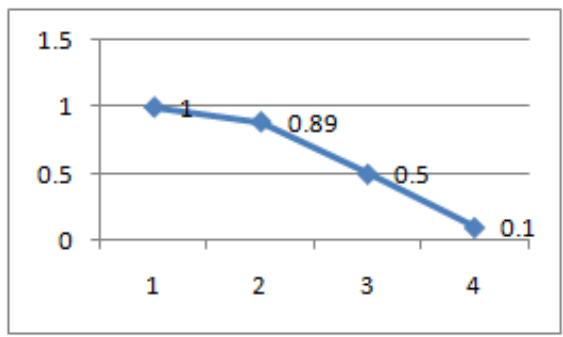

Fig.9 magnetic field lag time

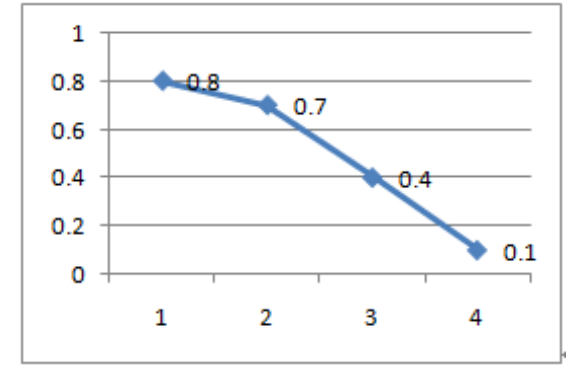

Fig.8 magnetic field value

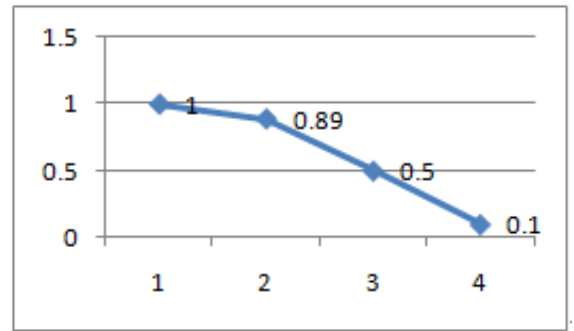

Fig.10 magnetic field lag time

\section{Arc Test Study}

The arc characteristics of the designed coil AMF contact were analyzed through experiments, and the characteristics of the magnetic field generated by this contact structure for arc control were verified based on the experimental results. A high current (40 kA) arc test was conducted on the new coil contact structure and the arcing time was set to $9 \mathrm{~ms}$. The arc pattern can reflect the arc control capability of the magnetic field. The arc test is carried out in a demountable arc chamber. The arc pattern is shown in Fig. 10. The clearance between open contacts is $55 \mathrm{~mm}$, the contact diameter is $100 \mathrm{~mm}$, the breaking current is $40 \mathrm{kA}$, and the contact structure breaking arc time was $9.8 \mathrm{~ms}$, and the peak current was $51.4 \mathrm{kA}$. The arc pattern of the contact structure at different breaking moments was analyzed. 


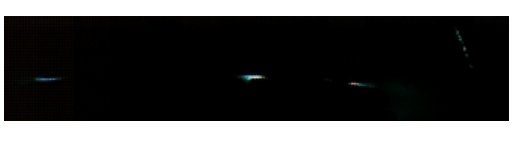

the first break $100 \mu \mathrm{s}$

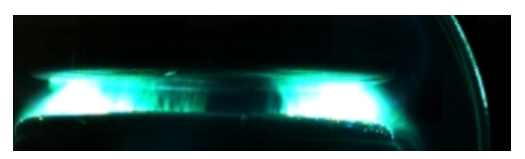

the first current peak

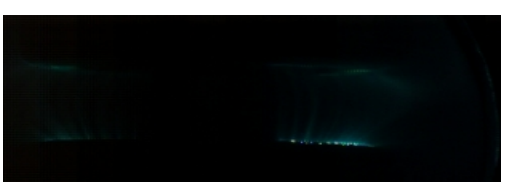

the first current 0 crossing

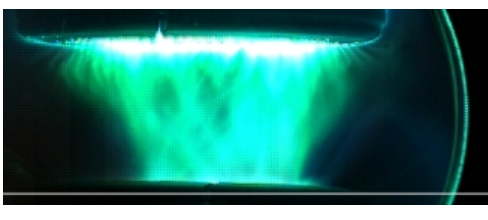

the second current peak

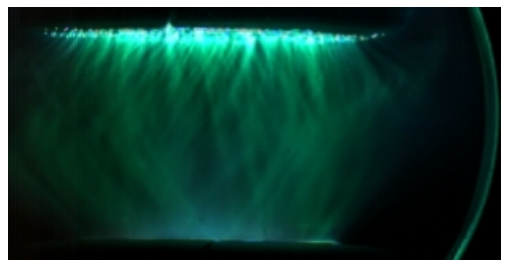

the second current 0 crossing

Fig.11 Comparative analysis of arc characteristics of two electrode structures It can be seen from FIG. 11 that the contact structure conducts research and analysis of arc patterns after the first break $100 \mu \mathrm{s}$, the first current peak, the first current zero crossing, the second current peak, and the second current zero crossing. According to the research and analysis, it can be seen from Fig. 11(1) that when the contacts are just separated, the arc is spotted and appears in three positions; 11(2) When the current reaches the first current half-wave peak, two arcs appear, the arc cathode appear bright spots, and the arc was severely agglomerated, in the strong arc mode, no visible metal droplets were splashed at this time, indicating that the vacuum arc does not severely ablate the contact structure at this time. It can be seen in Figure 11 (3) when the current reaches the first current zero crossing point, the arc between the contactsis in diffuse mode, no metal particles between the contact spatter, all arcs in the contact structure can be effectively controlled by the longitudinal magnetic field. It can be seen in Fig. 11(4) that when the current reaches the second current peak, the arc anode has formed a spot, the jet flow has not yet formed, and the cathode is in a diffusion state. Figure 11(5) can be seen when the current reaches the second current zero crossing, the arc is indiffuse mode, and the energy is small.

In the new coil structure, the first current peak shape in the breaking process and the arc burning phenomenon when the first current crosses zero are two arcs. Through analysis, the distribution of the magnetic field on the contact chip is shown in Figure 100. There are two places where the magnetic field is strong on the contact chip, the magnetic field distribution is not uniform, and the cathode spot moves to the area where the longitudinal magnetic field is relatively large.

In the new coil structure, the first current peak shape in the breaking process, and the arcing at the first current zero crossing are two arcs. Through analysis, the distribution of the magnetic field on the contact chip is shown in Figure 12. There are two strong magnetic fields on the contact chip, the magnetic field distribution is not uniform, and the cathode spot moves to the area where the longitudinal magnetic field is relatively large.
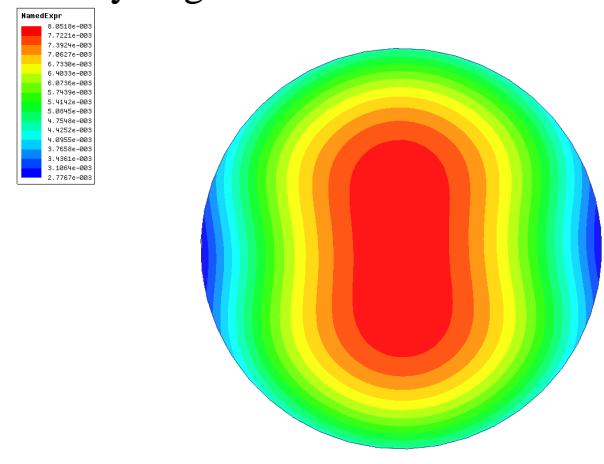

Fig.12 the distribution of the magnetic field on the contact chip 


\section{Break test}

Through magnetic field and 40kA arc test of the coil contact, the magnetic field generated by the new coil contact structure can effectively control the arc shape, convert the arc rapidly into a diffusion state, and facilitate the breaking. Therefore, a new type of contact structure can be used to further verify its performance. After $126 \mathrm{kV}$ vacuum interrupter technology, process and other aspects of research, the $126 \mathrm{kV}$ vacuum interrupter was successfully trial-produced and verifiedby experiments.
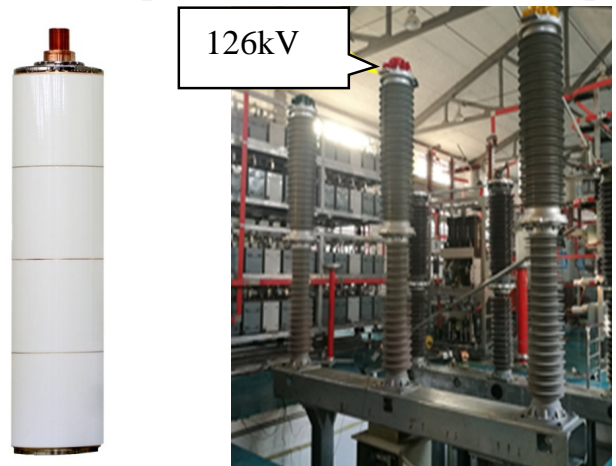

Fig.13 vacuum interrupter and vacuum circuit breaker

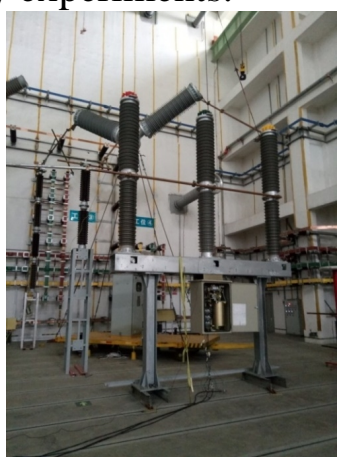

Fig.14 Breaking test

The $126 \mathrm{kV}$ vacuum interrupter adopts 4 sections of ceramic shells, adopts a new type of longitudinal magnetic field contact structure, and the main shield is embedded in a design scheme with a diameter of $208 \mathrm{~mm}$ and a total height of $920 \mathrm{~mm}$. The factory tests included resistance (applied pressure $6000 \mathrm{~N}$, $28 \mu \Omega)$, pressure resistance $(255 \mathrm{kV} / 1 \mathrm{~min})$, lightning impulse $(550 \mathrm{kV}, 15$ times each). Assemble the $126 \mathrm{kV}$ vacuum circuit breaker independently researched and developed by our company, is shown in Figure 13.

This test verifies that the JB/T 8738-2008 high-voltage AC switch equipment is executed with the vacuum interrupter standard and a 40kA high-current breaking test is completed at one time, is shown in Figure 14. Including T10, T30, T60, T100a9 (DC component 48\%), T100s. Accumulatively completed 20 times rated short-circuit current breaking.

As shown in Figure 15, The vacuum interrupter in the test verification was anatomically analyzed. The ablation of the contacts was as shown in the figure below. The contact piece keeps its original shape with almost no distortion. Seen from the front of the contact piece, the surface of the contact burned slightly and evenly without obvious deformation of the contact.

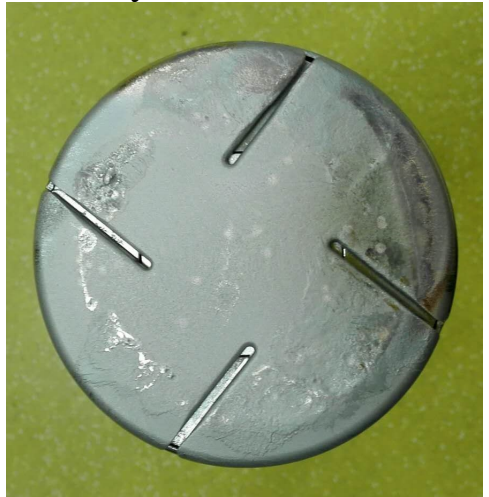

The surface of a movable contact

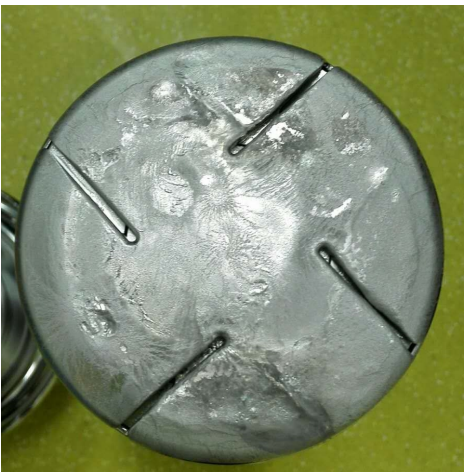

The surface of the static contact

Fig. 15 contact surface condition

\section{Conclusions}

In this paper, a new of AMF coil type contact structure for $126 \mathrm{kV}$ vacuum interrupter is designed. The magnetic field and arc of the contact structure were studied and verified by experiments. The following conclusions can be drawn:

1) through simulation calculation, the magnetic field intensity generated by the new contact structure decreases gradually from the middle to the edge, and the magnetic field is uniform. The 
magnetic field intensity, residual magnetic field, and lag time are basically consistent with the measurement results, and the simulation results are correct. The magnetic field generated by the contact structure meets the performance requirements

2) through the study of arc shape, the new coil structure can effectively control arc and transform arc into diffusion state rapidly. During the whole process from arc starting to arc extinguishing, no metallic droplets are sprayed, which is more conducive to breaking

3) A 40kA high-current breaking was performed 20 times with $126 \mathrm{kV}$ vacuum interrupter with a new type of contact structure, which verified the breaking capacity of the new coil structure. In terms of the breaking performance alone, it fully meets the breaking demand of $126 \mathrm{kV} / 40 \mathrm{kA}$.

\section{References}

[1] Liu Zhiyuan, Wang Zhongyi, Zhang Xuan ,Wang Jimei. Analysis of Axial Magnetic Field Characteristics of Coil Type Axial Magnetic Field Vacuum Interrupters [J]. Transactions of china electrotechnical society, 2007, 22 (1):47- 53.

[2] Hao Jiancheng, Yang Jiaxiang, Wang Xinzhang, ShangGuan Xianan. Simulation of 3-D Magnetic Field in two segments coil axial magnetic contact of $126 \mathrm{kV}$ vacuum interrupter[J].Procedings of the CSEE,2007(18):83-88.

[3] Zhiyuan Liu, Yingsan Geng, Jimei Wang. Technical development of high voltage vacuum circuit breaker[J]. Electric Age, 2008, 1:80-84.

[4] Lin Xin,Li Xintao,Li Luwei. Research Progress of Environment-friendly SF6 Substitution Media in electrical equipments[J].High Voltage Apparatus ,2016,52(12):1-7

[5] Jin Li, Study of high voltage and high interrupting capacity vacuum interrupter[D].shanxi: Xi'an Jiaotong University ,1997(in China).

[6] Zhongyi Wang, Zhiyuan Liu, Jimei Wang. Comparison of axial magnetic field characteristics of 5 axial magnetic field vacuum interrupter contacts $[\mathrm{J}]$. Advanced Technology of Electrical Engineering and Energy,2006,25(7): 21- 26.

[7] S.Yanabu, Y.Satoh, T.Tamagawa, E.Kaneko, S.Sohma,'Ten Year Experiment in AxialMagnetic Field-Type Vacuum Interrupters", IEEE Transaction on Power Delivery,Vol.PWRD-1,No.4,October 1986,pp:202-208.

[8] A.M. Chaly, A.A. Logatchev, K.K. Zabello, and S.M. Shkol'nik,"High current vacuum arc appearance in nonhomogeneous axialmagnetic field," IEEE Trans. Plasma Sci. vol.31, no.5, pp.884-889,Oct. 2003.

[9] Liu Zhiyuan, Xie Kesong,Wang Zhongyi, et al. Analysis of 3D eddy current field in cup type axial magnetic field vacuum Interrupter [ J] Adv. Tech. of Elec. Eng. \& Energy , 2004, 23( 2) : 26-28

[10] ZHANG Xuemin, WANG Xiaoqin, LIU Yixiong, Magnetic Field Characteristics and Arc Behaviors Research of 3/4 Coil-type AMF Contact for $126 \mathrm{kV}$ Vacuum Interrupter [J].High Voltage Apparatus ,2017,53(1):1-5 